\section{Future of UK observatory}

SIR-We are the group of astronomers of the Royal Greenwich Observatory (RGO) who are most directly responsible for the scientific care of the Anglo-Dutch telescopes on La Palma and we want to comment on the proposed move of RGO to the site of the Royal Observatory in Edinburgh (ROE), to the University of Cambridge or to the University of Manchester. Such a move would, at a crucial time, divert resources from the La Palma programme, from astronomy and from science, for benefits that are acknowledged to be intangible; we are opposed to a move of RGO.

However, given the assumption that the Science and Engineering Research Council (SERC) is now committed to move RGO, we believe that the council has an opportunity for a fresh approach to the organization of government astronomy in the United Kingdom. We hope SERC will build an institute which looks to the future of British optical astronomy. We hope that SERC will not, effectively, merge two government establishments in a way that perpetuates the existing way of working, the very mode which has been called into question. We see clearly that there are advantages to British astronomy of relocating $\mathrm{RGO}$ into an intellectual atmosphere, truly onto a university campus, and we prefer Cambridge for three reasons.

(1) We are very concerned about the effect of any move on the 4.2-m Herschel Telescope programme. We view with alarm the loss of productivity that has already been a baleful effect of the relocation issue, with literally hundreds of thousands of pounds' worth of time lost in anxious discussion before the move is even welldefined. The Herschel Telescope is the most ambitious project in UK optical astronomy, and it is now vulnerable because relocation has been made an issue at this unfortunate time. The further RGO is moved from its present home, the higher the proportion of key staff who will drop out of the Herschel Telescope project before it is completed. Even when the telescope is completed, we know from our experience with the other La Palma telescopes how essential it is to retain continuity of engineering staff in maintenance and development. A move within southern England to Cambridge would minimize disruption for La Palma.

(2) We have strong links with our colleagues in the British, Dutch, Irish and Spanish universities. We are determined to maintain these links if the move goes ahead to whatever university campus. We want to retain RGO's identity as an independent establishment with a duty to run La Palma for the whole of the astronomical community with which we are proud to collaborate and which we are proud to serve. In order to do this effectively, we and our co-workers need to be able to attend meetings, to listen to what our colleagues have to say and to share our knowledge of La Palma with them. Our colleagues need access to the facilities with which we back up our overseas telescopes. It is important for RGO to be centrally placed in the United Kingdom, with easy access to and from Europe. Despite our present proximity to the major airports which serve Europe (and La Palma), and to motorways that connect us to our colleagues in Britain, some of our university colleagues think Sussex is inaccessible. It is inconceivable that a move to Edinburgh would improve RGO's accessibility for the broad astronomical community. Of the stated options, only Cambridge and Manchester are central enough to justify in this respect a move from Sussex.

(3) The reason that we exist as a group is to stimulate the building programme for $\mathrm{La}$ Palma instruments and the operation of La Palma telescopes from the point of view of astronomy. The move from Sussex would increase this stimulation only if the move were to an excellent university with the best and largest astronomy departments and the most active engineering and instrument science programmes. Cambridge offers the most direct access by the La Palma project to a broad spread of good relevant departments, including outstanding optical, radio and theoretical astronomy institutes. Cambridge is better for SERC's declared aim of improving the interaction between RGO and the universities because the astronomy, physics and engineering departments are closely grouped and near to the industrial science park.

If the move of RGO goes ahead, we strongly favour Cambridge over the other two possibilities that have been proposed. and we are strongly opposed to a move to Edinburgh.

Peter Andrews, Bob Argyle, Charles Jenkins, Derek Jones, Robert Laing. TOM Marsh, Bill Martin, Paul Murdin, Max Pettini, Neill Reid, Hans Schild, Bill Sparks, Keith Taylor. Roberto Terlevich, Keith Tritton, JASPER WALL, ROY WALlis

Royal Greenwich Observatory,

Hailsham, East Sussex, BN27 1RP, UK

\section{Freedom of thought}

SIR--We refer to recent correspondence from C.K. Pallaghy (Nature 320, 9; 1986) of the School of Biological Sciences of this university stating his views on the size of Noah's Ark. Detailed refutations of such statements and conclusions. offered in support of the creationist position of $\mathrm{Dr}$ Pallaghy and others, have been provided in many sources in recent years and we do not intend to canvass them yet again. We do not deny the right of a tenured academic to espouse whatever opinions he believes worthy of promulgation. through whatever extracurricular forum he is able to capture.

We do, however, dissociate ourselves absolutely from the creationist views expressed by Dr Pallaghy in Nature and elsewhere, and we wish it to be placed on record that Dr Pallaghy's creationist pronouncements are strictly his own personal opinions and are not part of our school's curricula. The other staff members of the School of Biological Sciences of La Trobe University remain totally committed to rational, scientific explanations of biological and all other natural phenomena.

I.W.B. THORNTON

P.A. PARSONS

B.A. STONE

J.S. WAID

A.B. WARDROP

School of Biological Sciences,

La Trobe University,

Bundoora, Victoria, Australia 3083

\section{Theoretical choices}

SIR-In his book review "Cortical convolutions" (Nature 320, 689; 1986), Colin Blakemore implies that I reject "pure empiricism" and hold that it would be desirable to have "prior agreement on a systematic and exhaustive exposition of all possible hypotheses". This is not quite right. I have, it is true, argued at length that "pure empiricism", in the form in which it is advocated by Popper, for example, is untenable. Scientific theories cannot be selected solely on the basis of empirical success and failure. just because this would not enable us to reject infinitely many grossly non-explanatory but empirically successful theories. In science, two criteria always govern choice of theory: (1) empirical success and failure, and (2) degree of "simplicity" or "explanatoriness". As the mature Einstein always emphasized, science cannot proceed without the assumption that the Universe is comprehensible. in some way or other. comprehensive or explanatory theories thus deserving more serious consideration than incomprehensive, non-explanatory theories (sometimes even in the teeth of empirical considerations).

None of this. however. implies that it is possible to give a "systematic and exhaustive exposition of all possible hypotheses“. In my view, in general. this is not possible.

Nicholas Maxwell

Department of History \& Philosophy

of Science.

University College London.

Gower Street, London WCIE 6BT, UK 